\title{
Compuestos importantes para la salud encontrados en los cereales enteros
}

\author{
Rafael Villanueva Flores \\ Universidad de Lima. Lima, Perú \\ Correo electrónico: RVillan@correo.ulima.edu.pe
}

Recibido: 9/12/2011 / Aprobado: 4/4/2012

\begin{abstract}
RESUMEN: Los productos basados en granos enteros han ganado importancia, pues se les considera entre los alimentos que mejoran la salud y reducen el riesgo de enfermedades relacionadas con los hábitos alimentarios. La cáscara y el germen de los cereales contienen más vitaminas, minerales, antioxidantes naturales y fibra dietética que el endosperma, que principalmente contiene almidón y proteína. Estudios recientes indican que existen una serie de beneficios para la salud relacionados con el consumo de fibra dietética, entre los cuales se pueden mencionar la regulación de la glucosa de la sangre y del nivel de insulina, la reducción del colesterol de la sangre, la prevención del cáncer de colon y de desórdenes cardiovasculares.
\end{abstract}

Palabras clave: Cereales / cáscara / salvado / salud / prevención de enfermedades

\section{Health important compounds found in whole grains}

AвSTRACT: Whole grain based products are gaining importance in improving health and reducing risk diseases related to food habits. Cereal bran and germ contain more vitamins, minerals, natural antioxidants and dietary fiber than the endosperm that mainly contains starch and protein. Actual research shows beneficial aspects for health related to the consumption of dietary fiber like, for example, blood glucose and insulin level regulation improvement, blood cholesterol reduction and colonic cancer and cardiovascular disorders prevention.

Keywords: Cereals / bran / health / prevention 


\section{INTRODUCCIÓN}

Los cereales constituyen la fuente principal de alimentos para el mundo. Cuando se consumen tras quitarle las cubiertas y el germen, se denomina cereal 'refinado'. Cuando se procesa sin quitarle las cubiertas, el producto resultante se denomina 'entero'.

En los últimos años, los productos basados en granos enteros han ganado atención debido a los beneficios que aportan a la salud. Podemos definir a los granos enteros como aquellos granos intactos, molidos, chancados o laminados luego de haberles removido las partes no comestibles, en los cuales sus principales componentes (endosperma, germen y cáscara) mantienen la misma proporción relativa, tal como existen en el grano intacto. Se consideran también aquellos que sufrieron la separación temporal de sus componentes durante el procesamiento para una posterior recombinación.

Cada día es más evidente que los productos elaborados sobre la base de granos enteros tienen potencial para mejorar la salud y para reducir el riesgo de enfermedades relacionadas con los hábitos alimentarios.

Los cereales son principalmente fuente de carbohidratos provenientes del endosperma del grano de donde se obtiene la mayoría de los productos industriales, como la harina de trigo o el arroz pilado. Sin embargo, también son una fuente importante de componentes favorables para la salud, como la fibra dietética, los prebióticos, los minerales, los lípidos, las vitaminas y las ligninas ubicados principalmente en la cáscara y el germen del grano que, en muchos casos, al ser subproductos de los procesos de molienda, se destinan para la alimentación animal.

\section{LOS CEREALES}

\subsection{Los carbohidratos}

Los cereales son, principalmente, una fuente de carbohidratos con elevado contenido de almidón y fibra dietética y bajo contenido de azúcares simples. Aproximadamente, el $87 \%$ de grano del centeno y el $80 \%$ del grano de la avena consisten de endosperma comparado con el 8184\% para el caso del trigo (Nyström et al., 2007).

El almidón está concentrado en el endosperma, que es el depósito alimenticio de la planta. Durante la molienda, se aprecia un aumento 
importante en la fibra dietética con extracciones ${ }^{1}$ por encima del $80 \%$. Sin embargo, la cáscara y el germen también contienen la mayor parte de las vitaminas, los minerales y un rango de otros componentes bioactivos del grano.

\subsection{Fibra dietética}

La fibra dietética es la parte comestible de las plantas o hidratos de carbono análogos que son resistentes a la digestión y absorción en el intestino delgado, con fermentación completa o parcial en el intestino grueso (AACC International, 2001).

Los granos enteros son una fuente importante de fibra dietética, y la fibra de granos, como la del trigo, la avena, la cebada y el centeno aumentan el peso de las heces, aceleran el tránsito intestinal, fermentan en ácidos grasos de cadenas cortas y modifican la microflora del intestino (Slavin, 2010). El contenido de fibra dietética en los cereales varía de $4 \%$ en el arroz a $15 \%$ en el centeno. Dentro del grano, los polisacáridos sin almidón, ${ }^{2}$ el almidón resistente ${ }^{3}$ y los oligosacáridos son los mayores contribuyentes de la fibra dietética total. La mayor parte del contenido de fibra dietética disminuye considerablemente del pericarpio exterior al endosperma, con excepción del arabinoxilano, que es un componente importante de los materiales de las células de la pared del endosperma.

Los polisacáridos no celulósicos de las paredes de las células de los cereales no son digeridos por las enzimas del intestino delgado y, por ello, contribuyen a la ingesta de fibra dietética total. La efectividad de estos polisacáridos no celulósicos en la mejora de la salud está relacionada con su estructura fina y sus propiedades fisicoquímicas.

La fibra dietética, natural o añadida a los alimentos, tiene efectos favorables para la salud. La fibra dietética soluble influye en diferen-

1 En molinería, el porcentaje de harina y afrecho obtenido expresado como porcentaje de la cantidad de grano molido.

2 Heterogéneo grupo de carbohidratos originados en las paredes de las células de las plantas, que incluye celulosa, hemicelulosa, $\beta$-glucano, lignina y una porción menor de pectina.

3 Polímeros de glucosa que no pueden ser digeridos en el tracto gastrointestinal superior debido a la inaccesibilidad a la amiólisis, obstáculos físicos, retrogradación amiolítica o modificación química (Sajilata et al., 2006). 
tes elementos del síndrome metabólico y puede prevenir la diabetes tipo 2, el cáncer de colon y los desórdenes cardiovasculares (Galisteo et al., 2008; Collins et al., 2010). Esto ha sido explicado por los efectos viscosos de las fibras solubles, las cuales en la parte superior del intestino delgado pueden reducir o retrasar la absorción de carbohidratos y grasas. El efecto puede deberse al impedimento en el transporte de nutrientes a la superficie absorbida (Anderson et al., 2009; Brownlee, 2011) o por el retraso en el transporte de las enzimas digestivas a sus substratos (Schneeman, 1998), que resulta en una menor concentración de glucosa, insulina y colesterol en la sangre (Onning, 2004; Galisteo et al., 2008; Ulmius et al., 2009). La influencia directa de la fibra dietética en la salud del colon es mediada por las propiedades fermentativas y fisicoquímicas determinadas por la composición química y molecular de sus estructuras. La fibra dietética soluble (como el B-glucano) presenta una gran dispersión en agua, facilita la difusión de microbios y promueve la degradación de la fibra, que generalmente resulta en fermentabilidad elevada. Por contraste, la fibra dietética insoluble (como la celulosa y el arabinoxilano insoluble en agua) reducen el tiempo de tránsito de las heces en el colon debido a propiedades de retención de agua u aglomeración, pudiendo causar efectos laxativos favorables para aliviar el estreñimiento (Schneeman, 1998).

El B-glucano, una fibra soluble, se encuentra principalmente en la cebada (3-11\%), en la avena (3-7\%) y en el centeno (1-2.5\%). Esta fibra es un B-D-glucano $(1 \rightarrow 3)(1 \rightarrow 4)$ que presenta secciones de enlaces tipo celulosa unidos a través de enlaces glucosídicos $(1 \rightarrow 3)$, que permiten al B-glucano formar una solución viscosa luego de la solubilización. En general, el B-D-glucano $(1 \rightarrow 3)(1 \rightarrow 4)$ de los cereales está ubicado en la capa de aleurona ${ }^{4}$ y en las paredes de las células del endosperma (Ciu \& Wang, 2009).

La Agencia de Medicamentos y Alimentos de los Estados Unidos (FDA, por sus siglas en inglés) ha reconocido la eficacia del B-glucano, la mayor fuente de fibra dietética en la cebada, en la reducción del riesgo de problemas coronarios. En Estados Unidos, los productos a base de granos enteros de cebada pueden comercializarse con esta indicación (FDA, 2005). Similar situación se presenta en la Comunidad Europea (Bryngelsson \& Asp, 2007). Se pueden obtener productos de

4 Desde el punto de vista botánico, la capa de aleurona es la capa más externa del endosperma pero es removida durante la molienda y pasa a formar parte del afrecho. 
cebada con elevado contenido de fibra combinando diversos procesos de perlado, molienda, tamizado y clasificación por aire.

El arabinoxilano, una fibra insoluble, se encuentra en las células de las paredes de los cereales como trigo, cebada y centeno. El contenido promedio en la harina de trigo es de $2.1 \mathrm{~g} / 100 \mathrm{~g}$ (Andersson \& Aman, 2001). También se le encuentra en alta concentración en los subproductos del procesamiento del trigo. Su estructura es una columna lineal de $(1 \rightarrow 4)$ unida a residuos B-D-xilopiranose, substituido principalmente con a-L-arabinofuranosa en O-2 u O-3. Las propiedades físicas tales como la viscosidad, dependen del grado de substitución, la distribución de los substitutos y el nivel de polimerización (Haskå et al., 2010).

\subsection{Prebióticos}

En 1995, Gibson y Roberfroid definieron a los prebióticos como ingredientes no digeribles que afectan favorablemente al organismo mediante la estimulación selectiva del crecimiento o actividad de una o de un limitado número de bacterias en el colon, mejorando la salud. Esta definición fue actualizada por Gibson et al., (2004) y descrita como ingredientes selectivamente fermentables que permiten cambios específicos en la composición o actividad en la microbiota intestinal que mejora la salud. Los candidatos a prebióticos son: a inulina, fructo oligosacáridos, galacto oligosacáridos, soli oligosacáridos, xilo oligosacáridos, pirodextrinas e isomalto oligosacáridos (Mcafarlane et al., 2006).

El contenido de fructo oligosacáridos en el trigo es del 0.8-4.0\% en el material fresco (Venazza et al., 2006). En los cereales existen al menos dos oligosacáridos: galactosil y fructosil. Para el caso del trigo, los oligosacáridos se encuentran en la cáscara (Yamada et al., 1997) y en el germen (Pomeranz, 1998).

Los cereales también son fuente concentrada de almidón resistente, que ha sido descrito como un prebiótico emergente, con pruebas en animales pero con estudios limitados en humanos.

\subsection{Aminoácidos y péptidos}

Las células de la capa aleurona contienen aminoácidos esenciales (por ejemplo lisina), pero su disponibilidad puede estar limitada debido al espesor de las paredes de las células que las rodean. 
Recientemente, se ha descubierto que varias especies de cereales contienen el aminoácido 43 péptido denominado lunasina. Este péptido fue descubierto primero en la soya y posteriormente en la cebada, trigo y recientemente en el centeno coreano (Jeong et al., 2009). La lunasina es quimiopreventiva y se indica que tiene efectos en la reducción del colesterol (Gálvez, 2008). Comparado con otros cereales, la avena contiene el nivel más alto de proteína bien balanceada, con 18 aminoácidos, 8 de los cuales son balanceados (Lasztity, 1998).

La avena presenta un buen potencial para reducir el colesterol debido al contenido elevado de B-glucano en el grano entero (Wood, 2010). Adicional a ello, con una ingesta diaria de 10 gramos de 8 -glucano de avena, mejora la función gastrointestinal y el metabolismo de la glucosa (Malkki \& Virtanen, 2001).

\subsection{Minerales}

Los minerales están concentrados en las células exteriores del grano; las células de la capa de aleurona contienen hasta $60 \%$ del contenido de cenizas en el trigo y en los otros cereales. Consecuentemente, la harina integral tiene mayor contenido de minerales que las harinas refinadas. Sin embargo, la mayor parte (87\%) de los enlaces fítofosforosos están concentrados en las células de la capa de aleurona, lo cual limita la biodisponibilidad. La disminución en el contenido de minerales a niveles menores de extracción es menos dramática en la avena y en el centeno que en el trigo.

\subsection{Lípidos}

La mayoría de los cereales tienen un contenido graso entre 1,5\% y $4 \%$, pero el contenido en la avena es considerablemente más alto (3-9\%). Los lípidos están concentrados en el germen, pero también hay un contenido elevado en las células de la capa de aleurona (Fulcher \& Duke, 2002). La avena presenta una concentración elevada de lípidos en el endosperma, de manera que en contraste con otros cereales, en los cuales al retirar el germen con la cáscara durante el proceso de molienda se reduce considerablemente el contenido de grasa, este efecto es menos pronunciado en el caso de la avena. El ácido oleico representa el $30-40 \%$ de los lípidos en la avena pero solo el $15 \%$ en otros cereales. Por otro lado, la avena tiene una menor proporción de ácidos grasos poliin- 
saturados (principalmente ácido linoleico), que representan el 42-44\% de la grasa, mientras que en otros cereales representa el 63-70\%. Sin embargo, la avena también tiene una actividad elevada de la enzima lipasa, particularmente en la capa de aleurona, en los tejidos embrionarios y en las fracciones de harina cercanas a la cáscara (Ekstrand et al., 1992). Por ello es necesario un tratamiento hidrotérmico para prevenir la rancidez y el aumento de la vida útil. La harina integral es una buena alternativa de ingrediente funcional para el desarrollo de productos saludables.

\subsection{Vitaminas}

Los cereales son particularmente ricos en vitamina $\mathrm{B}$ como tiamina (B1), riboflavina (B2), niacina (B3), ácido pantoténico (B5), piridoxina (B6), biotina (B7) y ácido fólico. Las vitaminas están concentradas en las células de la capa de aleurona que contienen más del 80\% de la niacina en el trigo. El grano entero de centeno contiene ligeramente menos tiamina y bastante menos piridoxina y niacina que el grano entero de trigo, pero más riboflavina, folato y biotina. Cuando la extracción disminuye por debajo del 80\%, disminuye considerablemente en el contenido de vitamina $B$, pero esta reducción es menor en el centeno que en el trigo. En efecto, el endosperma del centeno contiene más riboflavina, folato, biotina y niacina que el endosperma del trigo.

La contribución de la vitamina B en la dieta con granos enteros es probablemente poco importante. En panes leudados con levadura, la levadura es un contribuyente mayor en la ingesta de vitamina B, la cual se estima en $70 \%$ del folato en el pan.

La vitamina $\mathrm{E}$ es importante debido a su efecto antioxidante contra la oxidación de grasas en las membranas biológicas, siendo el a-tocoferol el oxidante más efectivo. Sin embargo, estudios recientes han demostrado que el $\alpha$-tocotrienol es al menos tres veces más eficiente en interferir reacciones de cadenas con radicales libres (Panfili et al., 2003). El germen y la capa de aleurona son las principales fuentes de vitamina $\mathrm{E}$ en la forma de tocoferoles y tocotrienoles. El contenido de tocoles (unidad base de los tocoferoles) es comparable en los diferentes cereales, pero hay variaciones en el contenido de los diferentes isómeros. La avena y el centeno se caracterizan por tener un contenido mayor de a-tocotrienol, mientras que el trigo (suave y durum), espelta (especie de trigo) y triticale (híbrido entre el trigo y el centeno) tienen 
contenido elevado de B-tocotrienol. Esto también se refleja en el afrecho, donde los tocotrienoles están concentrados. El afrecho del centeno tiene un mayor contenido de a-tocotrienol que de B-tocotrienol, mientras que lo contrario sucede con el afrecho del trigo. Expresado como vitamina E equivalente, el contenido en la avena (34 mg/kg de DM) es aproximadamente 50\% mayor que en el trigo (23-24 mg/kg de DM) y que en el trigo duro y espelta ( 15 y $17 \mathrm{mg} / \mathrm{kg}$ de DM, respectivamente). No hay mucha información del contenido de tocoles en las diferentes fracciones de la molienda de cereales, pero desde que el germen y el afrecho se remueven, el contenido de vitamina $\mathrm{E}$ de una harina refinada es menor que en una harina integral. La cáscara del arroz, cebada, avena y el aceite de palma son las únicas fuentes naturales de altos niveles de vitamina E, principalmente en la forma de tocotrienoles (Ong, 1993; Sheppard et al., 1993; Souci et al., 2002).

\subsection{Fitoesteroles y estanoles}

El germen y las células de la capa de aleurona del grano también contienen fitoesteroles y estanoles, que tienen propiedades importantes en la reducción del colesterol (Nyström et al., 2007a). La harina integral de centeno y la harina integral de trigo contienen aproximadamente el doble de esteroles que la harina blanca y el afrecho contiene de dos a seis veces más que la harina integral. En la molienda del centeno, el cambio en el contenido es menos pronunciado con el cambio en el grado de extracción, mientras que en el grano de trigo hay una clara gradiente de aumento de esteroles próximo a las capas exteriores del grano (Nyström et al., 2007b). Esto está probablemente relacionado con una pobre separación durante el proceso de molienda. Los esteroles en los cereales están principalmente en las formas insaturadas de sitosterol y campasterol y las formas saturadas, sitostanol y campestanol, a pesar de que el brasicasterol es el componente dominante en el afrecho del trigo. Los esteroles se encuentran en formas libres o unidos con glucósidos de esterol o ferulato de esterol. Pese al menor contenido de estanoles, en comparación con las semillas oleaginosas, los cereales también son una fuente importante para la ingesta diaria.

\subsection{Betaína y cloro}

Los cereales contienen más cloro que betaína. La betaína (trimetilglicina) protege a una variedad de células del estrés osmótico y previene 
un nivel elevado de homocisteína, un factor de riesgo de enfermedades crónicas vasculares. El cloro oxida a la betaína en el hígado y en el riñón pero también al cloroacetilo y al diclorofosfato, que son esenciales para el funcionamiento normal de las células. Al aumentar el grado de extracción en la molienda del trigo aumenta tanto el contenido de betaína como el de cloro. Hay, por lo menos, cuatro veces más betaína y el doble de cloro en el grano completo que en la harina refinada, y casi tres veces más en la cáscara del grano que en el grano entero, lo cual indica que ambos componentes están localizados en la cáscara. El grano entero del centeno y el afrecho del centeno contienen niveles similares al trigo. El grano entero de la avena, por otro lado, contiene únicamente un décimo del nivel encontrado en el centeno y el trigo, y el contenido en la cáscara de la avena es solamente de $40 \%$ a $70 \%$ mayor que en el grano, respectivamente. La cáscara del trigo blanco contiene ligeramente más betaína que el germen y el contenido de cloro en el germen es el doble que en la cáscara.

\subsection{Lignina}

Las ligninas son componentes difenólicos que actúan como defensores naturales de las plantas (metabolismo secundario). En el intestino largo, las ligninas de las plantas se trasforman por medio de la microflora en enteroligninas (enterodiol y enterolactona), que son absorbidas y entran al torrente sanguíneo. Las ligninas son biológicamente activas y pueden proteger de algunas enfermedades como ciertos cánceres (mama, próstata, colon) y enfermedades cardiovasculares (Hallmans et al., 2003). Más de veinte ligninas de plantas se han identificado en la cáscara de diferentes cereales. El centeno es el cereal con el mayor contenido de lignina, tanto en el grano entero como en la cáscara. El contenido más elevado de lignina se encuentra en el pericarpio, seguido por la capa de aleurona y el menor contenido en el endosperma. Por ende, el contenido de ligninas en el centeno es de solo el 30\% del contenido en grano entero. Los granos enteros de avena tienen un mayor contenido que el grano entero de trigo, pero el contenido en la cáscara del trigo es mayor que en la cáscara de la avena.

\subsection{Avenantramidas}

La avena es el único cereal que contiene avenantramidas, las cuales son metabolitos con potentes propiedades antioxidantes (Chen et al., 
2007, Bratt et al., 2003) y rol potencial en la prevención de inflamaciones en las arterias, el desarrollo de arteriosclerosis y la inhibición de la proliferación de células cancerosas en el colon (Nie et al., 2007).

Las fracciones de la avena obtenidas por perlado, indican que las avenantramidas están concentradas en la capa de aleurona, pero se pueden encontrar concentraciones de casi el doble en los copos de avena cuando se comparan con la cáscara de la avena.

\subsection{Alquilrescinoles}

Los alquilrescinoles son lípidos fenólicos presentes en las partes externas de ciertos cereales con propiedades antioxidantes y la posibilidad de tener efectos anticáncer y antimicrobiano (Ross et al., 2003). Los alquilrescinoles se encuentran en mayor cantidad en el centeno que en el trigo y casi nada en la avena y en el arroz. Se ubican exclusivamente en el pericarpio y en la capa de aleurona. El fraccionamiento en el centeno muestra casi el doble de concentración en el pericarpio y el cuádruple en la capa de aleurona en comparación con el grano entero. Poseen diferentes cadenas de diferentes longitudes con número impar de átomos de carbono.

\subsection{Otros compuestos fenólicos}

En general, la cáscara de los cereales tiene un contenido elevado de compuestos antioxidantes que están concentrados principalmente en la capa de aleurona del grano. Los derivados de ácido benzoico y los derivados del ácido cinámico al igual que los flavonoides están localizados aquí, pero el contenido y composición de los fenólicos en las células de las capas exteriores del grano son muy diferentes. La importancia biológica no está completamente entendida, pero parece estar relacionada con la capacidad antioxidante que correlaciona con el contenido de compuestos fenólicos.

Los flavonoides se presentan en las plantas como glucósidos en donde un grupo de azúcar (glicona,) se une covalentemente a otro grupo (libre de azúcar llamado aglicona) mediante enlaces O-glucosídico o C-glucosídico (Hirawan \& Beta, 2011). Los flavonoides, incluidos los 
flavones, están concentrados en la cáscara y germen del grano de trigo (Adom et al., 2005). El mecanismo antioxidativo de los flavonoides incluye la generación de radicales flavonoides menos reactivos debido a un menor potencial redox, previniendo así la oxidación de grasas (Cos et al., 2011).

\section{CONCLUSIONES}

La cáscara y el germen de los granos de cereales contienen más vitaminas, minerales, antioxidantes naturales y fibra dietética que el endosperma, el cual, principalmente, contiene almidón y proteína. Los productos hechos de granos enteros son más saludables que los productos refinados, dado que proporcionan la fibra dietética, almidón resistente y oligosacáridos necesarios para la dieta.

$\mathrm{Al}$ igual que la fibra dietética, la mayoría de vitaminas, minerales, cierto rango de esteroles, fenoles y otras sustancias bioactivas se encuentran en el germen, en la cáscara y en las capas externas del endosperma. Consecuentemente, el contenido de estos elementos se reduce significativamente en harinas con extracciones bajas cuando se les compara con harinas integrales. Por ello, al moler y separar la cáscara de la parte interna del endosperma no solo se reduce la cantidad de fibra beneficiosa para la salud sino también la de un conjunto importante de nutrientes y sustancias bioactivas con efectos beneficiosos para la salud. Esto aplica a todos los cereales, pero dadas las diferencias tanto en su molienda como en su inherente contenido de sustancias bioactivas estos efectos pueden variar. Más aún, la avena y el centeno contienen sustancias bioactivas que son únicas o que se presentan en niveles más elevados que en el trigo.

\section{REFERENCIAS}

1. AACC International. (2001). Definition of dietary fiber. Cereal Foods World, 46, 112-125.

2. Adom, K., Sorrell, M. E. \& Liu, R. H. (2005). Phytochemicals and antioxidant activity of milled fractions of different wheat varieties. J. Agric. Chem., 53, 2297-2306. 
3. Anderson, J. W., Baird, P., Davis, Jr., R. H., Ferreri, S., Knudtson, M., Koraym, A., Waters,V. \& Williams, C. L. (2009.). Health benefits of dietary fiber. Nutr. Rev. 67, 188-205.

4. Anderson, R. \& Aman, P. (2001). Cereal arabinoxylan: Occurrence, structure and properties. En Advance Dietary Fibre Technology, (pp. 301-314). Oxford: Blackwell Science.

5. Asp, N. G., Poutanen, K., Richardson, D. P. \& Van der Kamp, J. W. (2010). Whole grain definition. The Healthgrain Consortium. Recuperado el 6 de diciembre de 2011, de http://www.healthgrain. org/webfm_send/44

6. Basman, A. \& Köksel, H. (1999). Properties and composition of Turkish flat bread (bazlama) supplemented with barley flour and wheat bran. Cereal Chem, 76, 506-511.

7. Bratt, K., Sunnerheim, K., Bryngelsson, S., Fagerlund, A., Engman, L., Andersson, R. E. \& Dimberg, L. H. (2003). Avenanthramides in oats (Avena sativa L.) and structure-antioxidant activity relationships. J. Agric. Food Chem, 51, 594-600.

8. Brownlee, I. A. (2011). The physiological roles of dietary fiber. Food Hydrocolloids, 25, 238-250.

9. Bryngelsson, S. \& Asp, N.-G. (2007). Health claims according to Article 13 of the EC Regulation: Suggested priorities with reference to the Swedish Code on health claims and emphasis on relevance. Scan. J. Food Nutr., 51, 127-136.

10. Chen, C. Y. O., Milbury, P. E., Collins, F. W. \& Blumberg, J. B. (2007). Avenanthramides are bioavailable and have antioxidant activity in humans after cute consumption of an enriched mixture from oats. J. Food Nutr., 137, 1375-1382.

11. Ciu, S. W. \& Wang, Q. (2009). Cell wall polysaccharides in cereals: Chemical structures and functional properties. Struc. Chem, 20, 291-297.

12. Collins, H., Burton, R. A., Topping, D. L., Liao M-L., Bacic, A. \& Fincher, G. B. (2010). Variability in fine structures of noncellulosic cell wall polysaccharides and nutrition. Cereal Chem., 87, 272282. 
13. Cos, P., Calomme, M., Sindambiwe, J., De Bruyne, T., Cimanga, K., Pieters, L., Vlietinck, A. J. \& Berghe, D. V. (2001). Cytoxicity and lipid peroxidation-inhibiting activity of flavonoids. Planta Medica, 67, 515-519.

14. Ekstrand, B., Gangby, L. \& Akesson G. (1992). Lipase activity in oats-distribution, $\mathrm{pH}$-dependence, and heat inactivation. Cereal Chem., 69, 379-381.

15. Food and Drug Administration (2005). FDA allows barley products to claim reduction in risk coronary heart disease. Recuperado el 6 de diciembre de 2011, de http://www.fda.gov/bbs/topics/ news/2005/NEW01287.html

16. Fulcher, R. G. \& Duke, T. K. R. (2002). Whole-grain structure and organization: Implications for nutritionist and processors. En Whole-grain foods in health and disease. L. Marquart, J. Salvin \& R. G. Fulcher. AACC International, Saint. Paul, MN, EE. UU.

17. Galisteo, M., Duarte, J. \& Zarzuelo, A. (2008). Effects of dietary fibers on disturbances clustered in the metabolic syndrome. $J$. Nutr. Biochem., 19, 71-84.

18. Gálvez, A. F. (2008). Products and methods using soy peptides to lower total and LDL cholesterol levels. U. S. Patent PCT/ US2007/078584.

19. Gibson, G. R. \& Roberfroid, M. (1995). Dietary modulation of the human colonic microbiota: Introducing the concept of prebiotics. J. Nutr., 125, 1401-1412.

20. Gibson, G. R., Probert, H. M., Van Loo, J., Rastall, R. A. \& Roberfroid, M. (2004). Dietary modulation of the human colonic microbiota: Updating the concept of prebiotics. Nutr. Res., 17, 259-275.

21. Guo, W., Wise, M. L., Collins, F. W. \& Meydani, M. (2008). Avenanthramides, polyphenols from oats, inhibit IL-1B-induced NF-r B activation in endothelial cells. Free Radical Bio. Med., 44, 415-429.

22. Hallmans, G., Zhang, J. X., Lundin, E., Stattin, P. R., Johanson, A., Hultén, K. Winkvist, A., Lenner, P., Åman, P., \& Adlercreutz, H. (2003). Rye, lignans and human health. Proc. Nutr. Soc., 62, 193-199. 
23. Haskå, L., Nyman. M., \& Anderson, R. (2010). Characterization of indigestible carbohydrates in various fractions from wheat processing. Cereal Chem., 87, 125-130.

24. Hirawan, R. \& Beta, T. (2011). C-Glycosyflavone and lignan diglucoside contents of commercial, regular, and whole-wheat spaghetti. Cereal Chem., 88, 344-348.

25. Jeong, H. J., Lee, J. R., Jeong, J. B., Park, J. H., Cheong, Y. K. \& Lumem, B. O. (2009). The cancer preventive seed peptide lunasin from rye is bioavailable and bioactive. Nutr.Cancer, 61, 680-686.

26. Lasztity, R. (1998). Oat-grain-A wonderful reservoir of natural nutrients and biologically active substances. Food Rev. Int., 14, 99-119.

27. Le Gall, M., Serena, A., Jorgensen, H., Theil, P. K. \& Knudsen, K. E. B. (2009). The role of whole-wheat grain and wheat and rye ingredients on the digestion and fermentation process in the gut-A model experiment with pigs. Br. J. Nutr., 102, 1590-1600.

28. Macfarlane, S., Macfarlane, G. T. \& Cummings, J. H. (2006). Review article: Prebiotics in the gastrointestinal tract. Aliment. Pharm. Ther., 24, 701-714.

29. Mälkki, Y. \& Virtanen, E. (2001). Gastrointestinal effects of oat bran and oat gum: A review. Leben. Wiss Technol., 34, 337-347.

30. Nie, L., Wise, M., Collins, F. W. \& Meydani, M. (2007). Inhibition of colonic cancer cell proliferation and COX2 by oats avenanthramides (Avns). Faseb J., 21, A102-A103.

31. Nyström, L., Paasonen, A., Lampi, A. M. \& Piironen, V. (2007). Total plant sterols, steryl ferulates, and steryl glycosides in milling fractions of wheat and rye. J. Cereal Sci., 45, 106-115.

32. Ong, A. (1993). Natural sources of tocotrienols in vitamin $E$ in health and disease. Nueva York: F. J. Packer, Ed.

33. Onning, G. (2004). The use of cereal beta-glucans to control diabetes and cardiovascular disease. En Functional Foods, Cardiovascular Disease and Diabetes (pp. 126-149). Cambridge: A. Amoldi, Ed. Woodhead Publishing. 
34. Panfili, G., Fratianni, A. \& Irano, M. (2003). Normal phase highperformance liquid chromatography method for the determination of tocopherols and tocotrienols in cereals. J. Agric. Food Chem., 51, 3940-3944.

35. Pedersen, B. \& Eggum, B. O. (1983). The influence of milling on the nutritive value of flour from cereal grains 1 rye. Plant Food Hum. Nutr., 32, 185-196.

36. Pedersen, B. \& Eggum, B. O. (1983). The influence of milling on the nutritive value of flour from cereal grains 2 wheat. Plant Food Hum. Nutr., 33, 51-61.

37. Pomeranz, Y. (1998). Chemical composition of kernel structures. En Wheat: chemistry and technology (pp. 91-158). Minnesota: Pomeranz. AACC International.

38. Ross, A. B., Sheperd, M. J., Schüpphaus, M., Sinclair, V., Alfaro B., Kamal-Eldin A. \& Åman, P. (2003). Alkylresorcinols in cereals and cereal products. J. Agric. Food Chem., 51, 4111-4118.

39. Sajilata, M. G., Singhal, R. S. \& Kulkarni, P. R. (2006). Resistant starch-A review. Compr. Rev. Food Sci. Food Safety, 5, 1-17.

40. Schneeman, B. O. (1998). Dietary fiber and gastrointestinal function. Nutr. Rev., 18, 625-632.

41. Sheppard, A., Pennington, J. A. T. \& Weihrauch, J. L. (1993). Analysis and distribution of vitamin $\mathrm{E}$ in vegetable oils and foods, in vitamin $\mathrm{E}$ in health and disease, F. J. Packer, Nueva York: Marcel Dekker.

42. Slavin, J. (2010). Whole grains and digestive health. Cereal Chem. 87, 292-296.

43. Souci, S. W., Fachmann, W. y Kraut, H. (2002). Food Composition and Nutrition Tables, Florida: Ed. CRC Press.

44. Ulmius, M., Johanson, A. \& Onning, G. (2009). The influence of dietary fiber source and gender on the postprandial glucose and lipid response in healthy subjects. Eur. J. Nutr. 48, 395-402.

45. Venazza, C. L., Rabiu, B. A. \& Gibson, G. (2006). Human colonic microbiology and the role of dietary intervention: Introduction to 
prebiotics. En Prebiotics Development and Application (pp. 1-28). West Sussex: G. Gibson y R. Rastall, Eds. John Wiley and Sons.

46. Yamada, H., Itoh, K., Morishita, Y. \& Taniguchi, H. (1993). Structure and properties of oligosaccharides from wheat bran. Cereal Foods World, 38, 490-492.

47. Wood, P. J. (2010). Oat and rye B-glucan: Properties and function. Cereal Chem., 87, 315-330. 